\title{
Nrf2 is a key factor in the reversal effect of curcumin on multidrug resistance in the HCT-8/5-Fu human colorectal cancer cell line
}

\author{
CHAO ZHANG ${ }^{1-3}$, LIAN-JUN HE ${ }^{4}$, HAI-ZHU YE ${ }^{4}$, DING-FENG LIU ${ }^{3}$, YI-BAO ZHU ${ }^{4}$, DONG-DONG MIAO ${ }^{3}$, \\ SHENG-PENG ZHANG ${ }^{1-3}$, YUN-YU CHEN ${ }^{1-3}$, YUAN-WEI JIA ${ }^{1,5}$, JIE SHEN ${ }^{1,2,5}$ and XIAO-PING LIU ${ }^{1-3}$ \\ ${ }^{1}$ Center of Drug Screening and Evaluation; ${ }^{2}$ Anhui Provincial Engineering Research Center for Polysaccharide Drugs; \\ ${ }^{3}$ School of Pharmacy; ${ }^{4}$ School of Clinical Medicine, Wannan Medical College; ${ }^{5}$ Anhui Provincial Center for Drug \\ Clinical Evaluation, The First Affiliated Hospital of Wannan Medical College, Wuhu, Anhui 241000, P.R. China
}

Received April 24,2018; Accepted September 17, 2018

DOI: $10.3892 / \mathrm{mmr} .2018 .9589$

\begin{abstract}
Multidrug resistance (MDR) is a major concern when using chemotherapy for the treatment of patients with colorectal cancer. MDR modulators are agents that can reverse MDR and, thus, enhance the chemosensitivity of tumor cells. The development of MDR modulators can improve the therapeutic efficacies of MDR in cancer. However, few effective MDR modulators have been identified so far. Curcumin has been reported to be an effective compound in the reversal of MDR in colorectal cancer cells. However, the mechanisms associated with the reversal effect of curcumin on MDR and its regulation of target factors in MDR cells remain to be fully elucidated. 3-(4,5-dimethyl-2-thiazol)-2,5-diphenyltetrazolium bromide assays, flow cytometer apoptosis assays as well as mRNA and protein expression assays were performed in the present study, and the results confirmed the reversal effect of curcumin on HCT-8/5-Fu cells and provided evidence that activated nuclear factor erythroid 2-related factor (Nrf2) deficiency induced by the curcumin altered the B-cell lymphoma 2 (Bcl-2) associated $\mathrm{X}$ protein/Bcl-2 expression ratio, which led to the induction of apoptosis in HCT-8/5-Fu cells. These results indicated that Nrf2 may have a functional in the reversal effect of curcumin and contribute, at least in part, to the outcomes of chemotherapy in patients with MDR.
\end{abstract}

\section{Introduction}

Colorectal cancer is the fifth most common type of cancer and is the leading cause of cancer-associated mortality in China (1). Chemotherapy is the main clinical therapeutic method for colorectal cancer in addition to surgery and

Correspondence to: Dr Xiao-Ping Liu, Center of Drug Screening and Evaluation, Wannan Medical College, 22 Wenchang West Road, Yijiang, Wuhu, Anhui 241000, P.R. China

E-mail: liuxiaoping@wnmc.edu.cn

Key words: nuclear factor erythroid 2-related factor, multidrug resistance, curcumin, colorectal cancer, apoptosis radiotherapy (2). However, the rapid development of chemoresistance in colorectal cancer cells, particularly in its multiple form as multidrug resistance (MDR), which can either be intrinsic or acquired, is a major obstacle to overcome for the successful treatment of colorectal cancer (3). Identifying effective MDR modulators that are able to reverse MDR and can be used in combination with anticancer drug therapies has been a promising strategy to increase the success rate of chemotherapy. However, a number of MDR reversal agents frequently exhibited unacceptable levels of toxicity when they were administered at an effective dose in clinical trials (4-6). These side effects have consequently limited their application in clinical settings. To overcome the MDR of colorectal cancer, it is essential to develop novel reversal compounds with higher efficacy and lower toxicity.

Curcumin is a natural product obtained from the dried rhizomes of Curcuma longa (turmeric), which has been used for centuries throughout Asian countries as a food additive, in cosmetics, and as a form of traditional Chinese medicine $(7,8)$. It exhibits a wide range of pharmacological properties, including anti-inflammatory, anti-angiogenic, anti-oxidative and anticancer effects (9-11). Previous studies have demonstrated that curcumin reversed MDR in various tumors, including gastric, hepatic, and cervical cancer $(12,13)$. However, the mechanisms associated with the MDR reversal effect of curcumin and its targets remain to be fully elucidated.

In the present study, the key target and mechanism underlying the reversal effect of curcumin were investigated using the MDR HCT-8/5-Fu human colorectal cancer cell line. The results indicated that nuclear factor erythroid 2-related factor (Nrf2) may be critical in the MDR reversal effect of curcumin by promoting the apoptosis of HCT-8/5-Fu cells. Together with previously published data, these results suggest that curcumin may be suitable for the clinical treatments used in patients with MDR.

\section{Materials and methods}

Cell culture. The HCT-8 human colorectal cancer cell line was purchased from the Type Culture Collection of the Chinese Academy of Sciences (Shanghai, China); the HCT-8/5-Fu cell line was purchased from the Advanced Research Center 
of Central South University (Changsha, China). The two cell lines were grown in RPMI-1640 medium supplemented with $10 \%$ (v/v) fetal bovine serum (FBS; Thermo Fisher Scientific, Inc., Waltham, MA, USA), and maintained in a humidified atmosphere with $5 \% \mathrm{CO}_{2}$ at $37^{\circ} \mathrm{C}$. Curcumin and 5-Fu were purchased from Sigma-Aldrich (Merck KGaA, Darmstadt, Germany). The HCT- 8 and HCT-8/5-Fu cells were treated with curcumin, $5-\mathrm{Fu}$, or curcumin combined with $5-\mathrm{Fu}$ for $24 \mathrm{~h}$ at $37^{\circ} \mathrm{C}$.

Cellviabilityassay. The effect of drug treatments on cell viability was quantified using the 3-(4,5-dimethyl-2-thiazol)-2,5-diphenyltetrazolium bromide (MTT) assay. Cells in the exponential growth phase were trypsinized and seeded in 96-well plates at an initial density of 5,000 cells/well and then treated with the scheduled treatment of drugs (for curcumin: set $100 \mu \mathrm{M}$ as the initial concentration, followed by 2 -fold dilution for 10 concentrations; for $5-\mathrm{Fu}$ : set $58.88 \mathrm{mM}$ as the initial concentration, followed by 2 -fold dilution for 13 concentrations). Complete RPMI-1640 medium without drugs was added to the blank wells. The control cells were treated with dimethyl sulfoxide (DMSO) only. Following incubation for 24 h, $10 \mu \mathrm{l}$ MTT (Amresco, Inc., Solon, OH, USA) was added to each well and the cells were incubated at $37^{\circ} \mathrm{C}$ for $4 \mathrm{~h}$. The medium was then carefully removed and $150 \mu 1$ DMSO was added, followed by gentle agitation. The optical density (OD) of the released color was read at $570 \mathrm{~nm}$. The cell growth inhibition rate was calculated using the following formula: Growth inhibition rate $=(\mathrm{OD}$ value of control $-\mathrm{OD}$ value of test $) /(\mathrm{OD}$ value of control - OD value of blank) x $100 \%$.

MDR reversal assay. A concentration of $10 \mu \mathrm{M}$ curcumin, which was lower than the $\mathrm{IC}_{10}$ value obtained following the MTT assay of curcumin alone in HCT-8/5-Fu cells, was designated as the reversal concentration of curcumin in the following experiments.

The HCT-8/5-FU cells were plated in 96-well plates at an initial density of 5,000 cells/well and allowed to grow for $12 \mathrm{~h}$. The cells were then pretreated with $10 \mu \mathrm{M}$ curcumin for $4 \mathrm{~h}$, followed by treatment with serial dilutions of 5-Fu for $24 \mathrm{~h}$ (set $58.88 \mathrm{mM}$ as the initial concentration, followed by 2 -fold dilution for 10 concentrations). The control cells were treated with 5-Fu alone. By performing the MTT assay, as described above, the $\mathrm{IC}_{50}$ of each treatment was calculated. The reversal fold change was defined with the following ratio: $\mathrm{IC}_{50}$ value of $5-\mathrm{Fu}$ alone/ $\mathrm{IC}_{50}$ value of 5-Fu combined with curcumin.

Apoptosis assay. The HCT-8/5-Fu cells were divided into the following four groups: i) No treatment group (control); ii) $10 \mu \mathrm{M}$ curcumin treatment group; iii) $10 \mathrm{mM} \mathrm{5-Fu} \mathrm{treat-}$ ment group; and iv) $10 \mu \mathrm{M}$ curcumin $+10 \mathrm{mM} 5-\mathrm{Fu}$ treatment group. All cells $\left(5 \times 10^{3}\right)$ were plated on 6-well plates for $12 \mathrm{~h}$, and then treated with curcumin or DMSO (control) for $4 \mathrm{~h}$, followed by 5 -Fu treatment for a further $24 \mathrm{~h}$. All cell cultures were performed at $37^{\circ} \mathrm{C}$ in an incubator containing $5 \% \mathrm{CO}_{2}$. The cells were then harvested, washed twice with ice-cold PBS, and evaluated for apoptosis using a FITC-Annexin V Apoptosis Detection kit I (BD Biosciences, San Jose, CA, USA) and analyzed using a Novocyte flow cytometer (ACEA Biosciences, Inc., San Diego, CA, USA) with NovoExpress
1.2.4 software (ACEA Biosciences, Inc.). Analysis of apoptotic rates was performed using GraphPad Prism 5.0 software (GraphPad Software, Inc., San Diego, CA, USA).

$R N A$ extraction and reverse transcription-quantitative polymerase chain reaction $(R T-q P C R)$ analysis. The drug treatments were performed as mentioned above. Total RNA was extracted using TRIzol reagent (Thermo Fisher Scientific, Inc.), and first strand cDNA was generated using the Reverse Transcription system (Takara Bio, Inc., Otsu, Japan; cat no. RR047A) in a $20 \mu \mathrm{l}$ reaction containing $1 \mu \mathrm{g}$ of total RNA. A $1 \mu 1$ aliquot of cDNA was amplified using the SYBR-Green PCR Master mix (Takara Bio, Inc.; cat. no. RR820A) in each $20 \mu \mathrm{l}$ reaction according to the manufacturer's instructions. The thermocycling conditions used for qPCR were as follows: $95^{\circ} \mathrm{C}$ for $10 \mathrm{~min}$; followed by 40 cycles at $95^{\circ} \mathrm{C}$ for $5 \mathrm{sec}, 60^{\circ} \mathrm{C}$ for $30 \mathrm{sec}$ and a 1 cycle dissociation stage at $95^{\circ} \mathrm{C}$ for $15 \mathrm{sec}$, $60^{\circ} \mathrm{C}$ for $1 \mathrm{~min}$ and $95^{\circ} \mathrm{C}$ for $15 \mathrm{sec}$. qPCR was performed using the ABI StepOne plus Real-Time PCR system with the following primers: Nrf2, forward 5'-ATAGCTGAGCCCAG TATC-3' and reverse 5'-CATGCACGTGAGTGCTCT-3'; Kelch-like ECH associated protein 1 (Keap1), forward 5'-CT GGTATCTGAAACCCGTCTA-3' and reverse 5'-TGGCTTCT AATGCCCTGA-3'; B-cell lymphoma 2 (Bcl-2), forward 5'-TT CTTTGAGTTCGGTGGGGTC-3' and reverse 5'-TGCATATT TGTTTGGGGCAGG-3'; Bcl-2-associated X protein (Bax), forward 5'-TCCACCAAGAAGCTGAGCGAG-3' and reverse 5'-GTCCAGCCCATGATGGTTCT-3'; and GAPDH, forward 5'-AGAAGGCTGGGGCTCATTTG-3' and reverse 5'-AG GGGCCATCCACAGTCTTC-3'. The relative expression values of Nrf2, Keap1, Bcl-2 and Bax were calculated and normalized to GAPDH in each sample using the $2^{-\Delta \Delta C q}$ method (14). The experiments were performed in triplicate.

Western blot analysis. The whole cells were lysed with RIPA lysis buffer (Beyotime Institute of Biotechnology, Haimen, China; cat. no. P0013B). Protein quantification was performed using a BCA Protein Assay kit (Thermo Fisher Scientific, Inc.; cat. no. 23225), following which equal quantities of proteins $(100 \mu \mathrm{g})$ were separated via a 4-20\% gradient SDS-PAGE gel, and transferred onto a PVDF membrane. The membranes were blocked with 5\% BSA (Merck KGaA) at room temperature for $2 \mathrm{~h}$. Antibodies against the following proteins were used: Rabbit anti-NRF2 was purchased from Abcam (Cambridge, MA, USA; cat. no. ab62352); rabbit anti-Keap1, rabbit anti-Bcl-2, rabbit anti-Bax and rabbit anti-actin were purchased from Cell Signaling Technology, Inc. (Danvers, MA, USA; cat. nos. 8047, 4223, 5023 and 8457, respectively). All primary antibodies were used at a 1:1,000 dilution in $5 \%$ BSA together with the membranes and incubated at $4^{\circ} \mathrm{C}$ overnight. Following three washes in TBS/0.1\% Tween-20, the membranes were each probed with a secondary horseradish peroxidase-conjugated goat anti-rabbit antibody (Abcam; cat. no. ab6721) at a 5,000-fold dilution in 5\% BSA. Following six washes with TBS/0.1\% Tween-20, the immune complexes were incubated with ECL reagents (Thermo Fisher Scientific, Inc.; cat. no. 34577), and detected using the ChemiDoc ${ }^{\mathrm{TM}}$ Touch Imaging System (Bio-Rad Laboratories, Inc., Hercules, CA, USA). The resulting bands on the membranes were calculated and normalized to $\beta$-actin in each sample using 

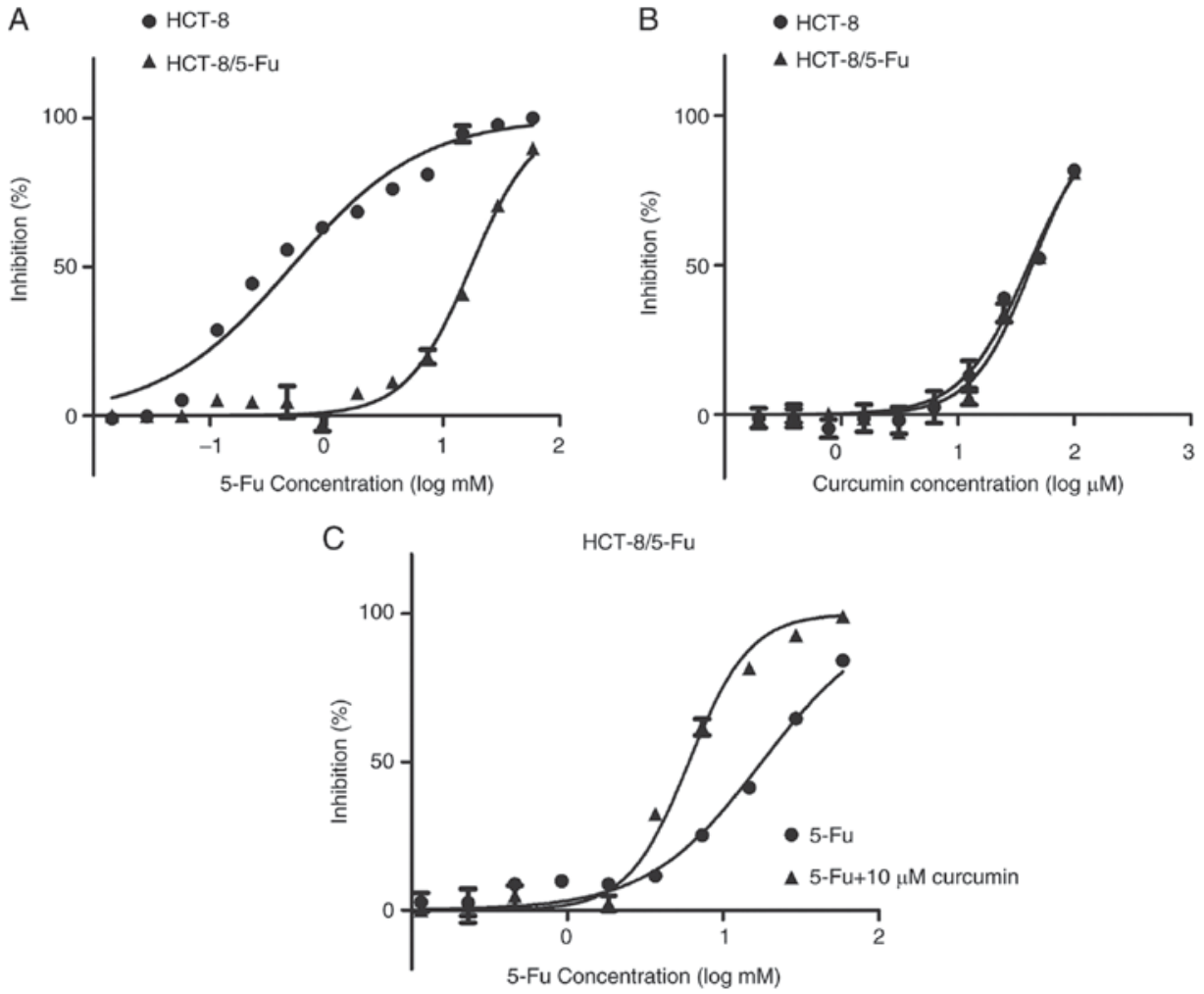

Figure 1. Curcumin enhances the chemosensitivity of HCT-8/5-Fu cells to 5-Fu. HCT-8 and HCT-8/5-Fu cells were treated with or without various concentrations of (A) 5-Fu or (B) curcumin. (C) HCT-8/5-Fu cells were treated with 5-Fu alone or in combination with curcumin, and the cell viability was measured using a 3-(4,5-dimethyl-2-thiazol)-2,5-diphenyltetrazolium bromide assay. Dimethyl sulfoxide was used as the negative control. The results are expressed as the mean \pm standard deviation $(\mathrm{n}=5)$ of three independent experiments.

ImageJ software (version 1.39u; National Institutes of Health, Bethesda, MD, USA).

Small interfering (si)-RNA and transfection. Nrf2 siRNA and a scramble non-targeting siRNA (siCtrl; negative control) were purchased from Biomics Biotechnologies Co., Ltd. (Nantong, China). The siRNA sequences were as follows: siNrf2, 5'-GA GACUACCAUGGUUCCAA-3'; and siCtrl, 5'-UUCUCCGA ACGUGUCACGU-3'. The siRNAs were transfected into cells using Lipofectamine $2000^{\circledR}$ reagent (Thermo Fisher Scientific, Inc.) according to the manufacturer's protocol.

Statistical analysis. The results are presented as the mean \pm standard deviation of at least three independent experiments for each group. Statistical differences were determined by one-way analysis of variance with Holm's post hoc test for multiple comparisons or two-sample t-tests for independent samples using SPSS 19.0 software (IBM SPSS, Armonk, IL, USA). Linear mixed effects models were used in analyses in order to account for the correlations among correlated observations, including cell growth measured over time in cell culture. $\mathrm{P}<0.05$ was considered to indicate a statistically significant difference.

\section{Results}

Curcumin enhances the chemosensitivity of HCT-8/5-Fu cells to $5-\mathrm{Fu}$. To validate the resistance to 5-Fu in HCT-8/5-Fu cells, the present study first examined the cytotoxicity of 5-Fu alone in the parental HCT-8 cells and MDR HCT-8/5-Fu cells using an MTT assay. In the HCT-8/5-Fu cells, a 36.90-fold increase in resistance to 5 -Fu was observed $\left(\mathrm{IC}_{50}\right.$ value: $17.53 \pm 0.2021$ $\mathrm{mM})$, compared with the parental HCT-8 cells $\left(\mathrm{IC}_{50}\right.$ value: $0.4751 \pm 0.02447 \mathrm{mM}$; Fig. 1A). The intrinsic in vitro toxicity of curcumin on HCT- 8 and HCT-8/5-Fu cells was also evaluated using the MTT assay. Curcumin inhibited the growth of the two cell types in a dose-dependent manner in vitro. The $\mathrm{IC}_{50}$ values of curcumin for the parental and resistant cells were $40.72 \pm 4.711$ and $43.81 \pm 2.116 \mu \mathrm{M}$ at $24 \mathrm{~h}$, respectively (Fig. 1B). The $\mathrm{IC}_{50}$ values of curcumin for the two cell lines were comparable, with no significant differences detected $(\mathrm{P}>0.05)$; this indicated that the HCT-8/5-Fu cells were not cross-resistant to curcumin. The $\mathrm{IC}_{10}$ value of curcumin for the HCT-8/5-Fu cells at $24 \mathrm{~h}$ was $\sim 12 \mu \mathrm{M}$ and, consequently, a concentration of $10 \mu \mathrm{M}$ was selected as the reversal concentration of curcumin for the subsequent experiments in the present study.

The reversal assay indicated that, for the HCT- $8 / 5$-Fu cells, the $\mathrm{IC}_{50}$ values of 5-Fu treatment alone and 5-Fu combined with $10 \mu \mathrm{M}$ curcumin at $24 \mathrm{~h}$ were $17.31 \pm 0.2325$ and $6.086 \pm 0.9890 \mathrm{mM}$, respectively (Fig. 1C). The reversal effects of $10 \mu \mathrm{M}$ curcumin on the HCT-8/5-Fu cells were 2.844-fold. Therefore, the results demonstrated that curcumin significantly increased the sensitivity of the HCT-8/5-Fu cells to 5-Fu.

Curcumin treatment combined with 5-Fu induces cell apoptosis in HCT-8/5-Fu cells. One mechanism by which MDR reversal agents enhance the sensitivity of MDR cells is via the induced apoptosis of these cells using MDR reversal agents (15-18). To investigate whether curcumin reverses the 


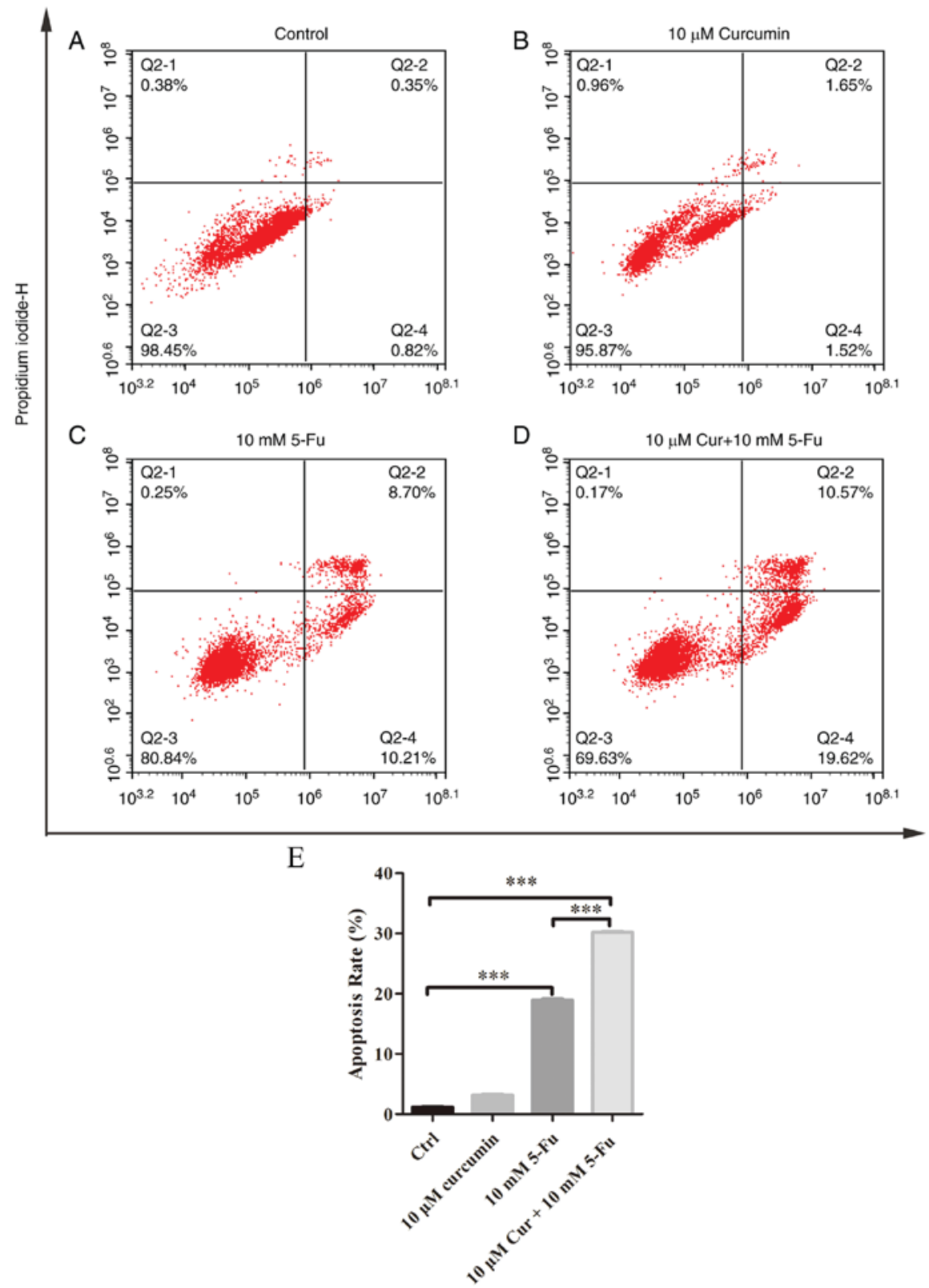

Figure 2. Curcumin combined with 5-Fu induces the apoptosis of HCT-8/5-Fu cells. HCT-8/5-Fu cells were treated with (A) dimethyl sulfoxide, (B) $10 \mu \mathrm{M}$ curcumin, (C) $10 \mathrm{mM}$ 5-Fu or (D) $10 \mu \mathrm{M}$ curcumin $+10 \mathrm{mM}$ 5-Fu for $24 \mathrm{~h}$. (E) The percentage of cell apoptosis was determined by Annexin V/propidium iodide staining and flow cytometry in three independent experiments and graphed by GraphPad Prism 5. ${ }^{* * * *} \mathrm{P}<0.005$. Cur, curcumin.

MDR of HCT-8/5-Fu cells by promoting apoptosis, the present study evaluated the levels of apoptosis in untreated HCT-8/5-Fu cells and in those treated with curcumin alone, 5-Fu alone, and curcumin combined with 5-Fu using flow cytometry. As shown in Fig. 2A-E, only a small number of cells underwent apoptosis following treatment with $10 \mu \mathrm{M}$ curcumin only $(3.17 \pm 0.13 \%$; $\mathrm{P}=0.0231$ ). Following $10 \mathrm{mM}$ of $5-\mathrm{Fu}$ treatment, the levels of apoptosis increased to $18.91 \pm 0.25 \%(\mathrm{P}=0.0117)$. However, the results also suggested that the combined treatment of 10 $\mu \mathrm{M}$ curcumin with $10 \mathrm{mM} 5$-Fu significantly increased the rate of apoptosis compared with 5-Fu only treatment in the HCT-8/5-Fu cells (apoptotic ratio: 30.19 $\pm 0.17 \%$; $\mathrm{P}=0.0092$ ). Taken together, these results indicated that the combined treatment with curcumin and 5-Fu induced HCT-8/5-Fu cell apoptosis, whereas curcumin treatment alone did not induce apoptosis.
Curcumin combined with 5-Fu downregulates the expression ratio of $\mathrm{Bax} / \mathrm{Bcl}-2$. To confirm the results of the flow cytometry, the expression levels of several genes involved in the apoptosis of HCT-8/5-Fu cells were quantified by RT-qPCR and western blot analyses. As shown in Fig. 3A, treatment with curcumin alone marginally increased the expression of Bax at the mRNA level compared with that in the untreated cells. However, treatment with 5-Fu alone or combined with curcumin upregulated the mRNA expression of Bax by 2- to 3-fold. By contrast, the mRNA expression of Bcl-2 was significantly upregulated following treatment with 5-Fu alone and downregulated following treatment with $5-\mathrm{Fu}$ in combination with curcumin compared with that in the untreated HCT-8/5-Fu cells $(\mathrm{P}<0.005)$. In addition, the expression ratio of $\mathrm{Bax} / \mathrm{Bcl}-2$ in cells treated with curcumin (ratio: 0.90 ) or 5-Fu alone (ratio: 1.02) was only marginally 

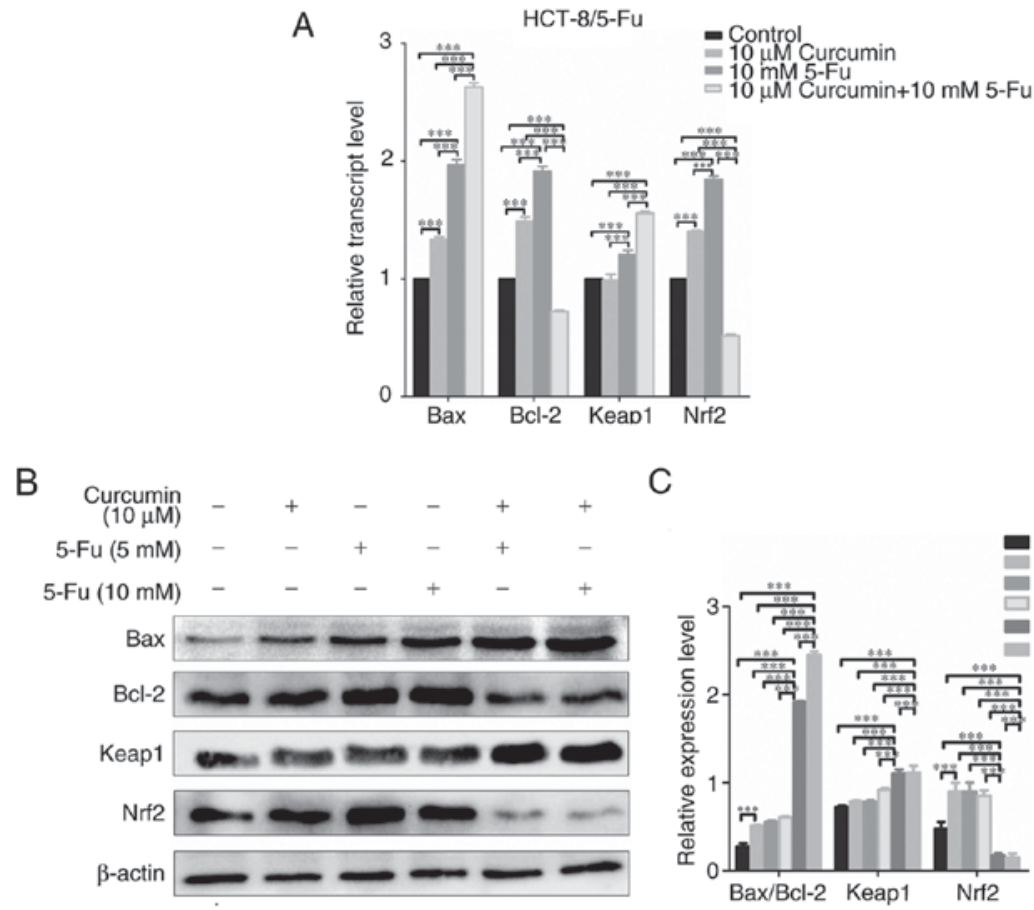

C

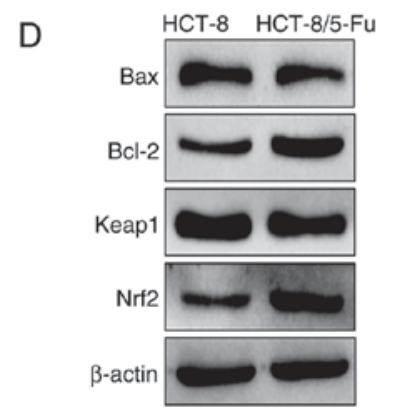

E

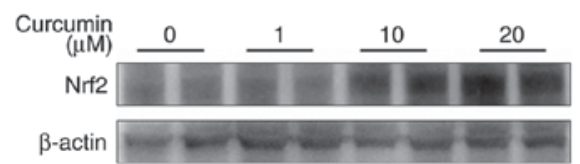

Figure 3. Curcumin combined with 5-Fu downregulates the downstream signal transduction of Nrf2 signaling. The effect on mRNA and protein expression levels of Bax, Bcl-2, Keap1 and Nrf2 were evaluated by (A) reverse transcription-quantitative polymerase chain reaction analysis or (B) western blot analysis, respectively. (C) Relative protein expression levels were quantified using ImageJ software and normalized to $\beta$-actin. ${ }^{* * *} \mathrm{P}<0.005$. (D) Expression levels of Nrf2 signaling-associated proteins in HCT-8 and HCT-8/5-Fu cells were determined. HCT-8/5-Fu cells were treated with DMSO, $10 \mu \mathrm{M}$ curcumin, and 5 or $10 \mathrm{mM}$ 5-Fu alone or in combination with $10 \mu \mathrm{M}$ curcumin for $24 \mathrm{~h}$. (E) HCT-8/5-Fu cells were treated with DMSO, $1 \mu \mathrm{M}$ curcumin, $10 \mu \mathrm{M}$ curcumin or $20 \mu \mathrm{M}$ curcumin for $24 \mathrm{~h}$. The protein expression was evaluated by western blot analysis. Data are expressed as the mean \pm standard deviation of three independent experiments. Nrf2, nuclear factor erythroid 2-related factor; Bcl-2, B-cell lymphoma 2; Bax, Bcl-2-associated X protein; Keap1, Kelch-like ECH associated protein 1; DMSO, dimethyl sulfoxide.

altered compared with that in the untreated HCT-8/5-Fu cells (ratio: 1.00); however, this ratio was markedly increased in the curcumin +5 -FU treatment group (ratio: 3.64 ). Similar results were obtained following western blot analysis of the protein levels (Fig. 3B and C). Together, these results indicated that treatment with curcumin alone did not alter the levels of apoptosis in HCT-8/5-Fu cells, as the expression ratio of Bax/Bcl-2 was relatively unchanged compared with that in normal HCT-8/5-Fu cells. However, HCT-8/5-Fu cells treated with curcumin and 5-Fu combined exhibited a markedly increased rate of apoptosis compared with that in normal cells due to the significantly increased expression ratio of $\mathrm{Bax} / \mathrm{Bcl}-2$.

Keapl/Nrf2 signaling may be involved in the MDR reversal effect of curcumin in HCT-8/5-Fu cells. Previous studies have demonstrated that there may be an important connection between cell apoptosis and the Nrf2-mediated regulation of proteins from the Bcl-2 family $(19,20)$. To investigate whether curcumin reversed MDR in HCT-8/5-Fu cells by modulating the Keap1/Nrf2 signaling pathway, the present study first examined the protein expression levels of Keap1, Nrf2, Bcl-2 and Bax in HCT- 8 and HCT-8/5-Fu cells. The results revealed that the expression levels of $\mathrm{Nrf} 2$ and $\mathrm{Bcl}-2$ were upregulated in HCT-8/5-Fu cells, however, the expression levels of Keap1 and Bax were downregulated (Fig. 3D). The expression of these proteins was then further evaluated at the mRNA and protein levels in HCT-8/5-Fu cells with or without treatment. As shown in Fig. 3A, the mRNA expression level of Keap1 was not altered in cells exposed to curcumin treatment alone compared with that in normal cells, whereas treatment with 5-Fu alone or together with curcumin significantly induced the mRNA expression of Keap1 $(\mathrm{P}<0.005)$. By contrast, the mRNA expression level of Nrf2 was markedly upregulated in cells treated with curcumin or 5-Fu only, compared with that in normal cells, whereas combined treatment with $5-\mathrm{Fu}$ and curcumin significantly suppressed the mRNA expression of Nrf2 $(\mathrm{P}<0.005)$. Similar 

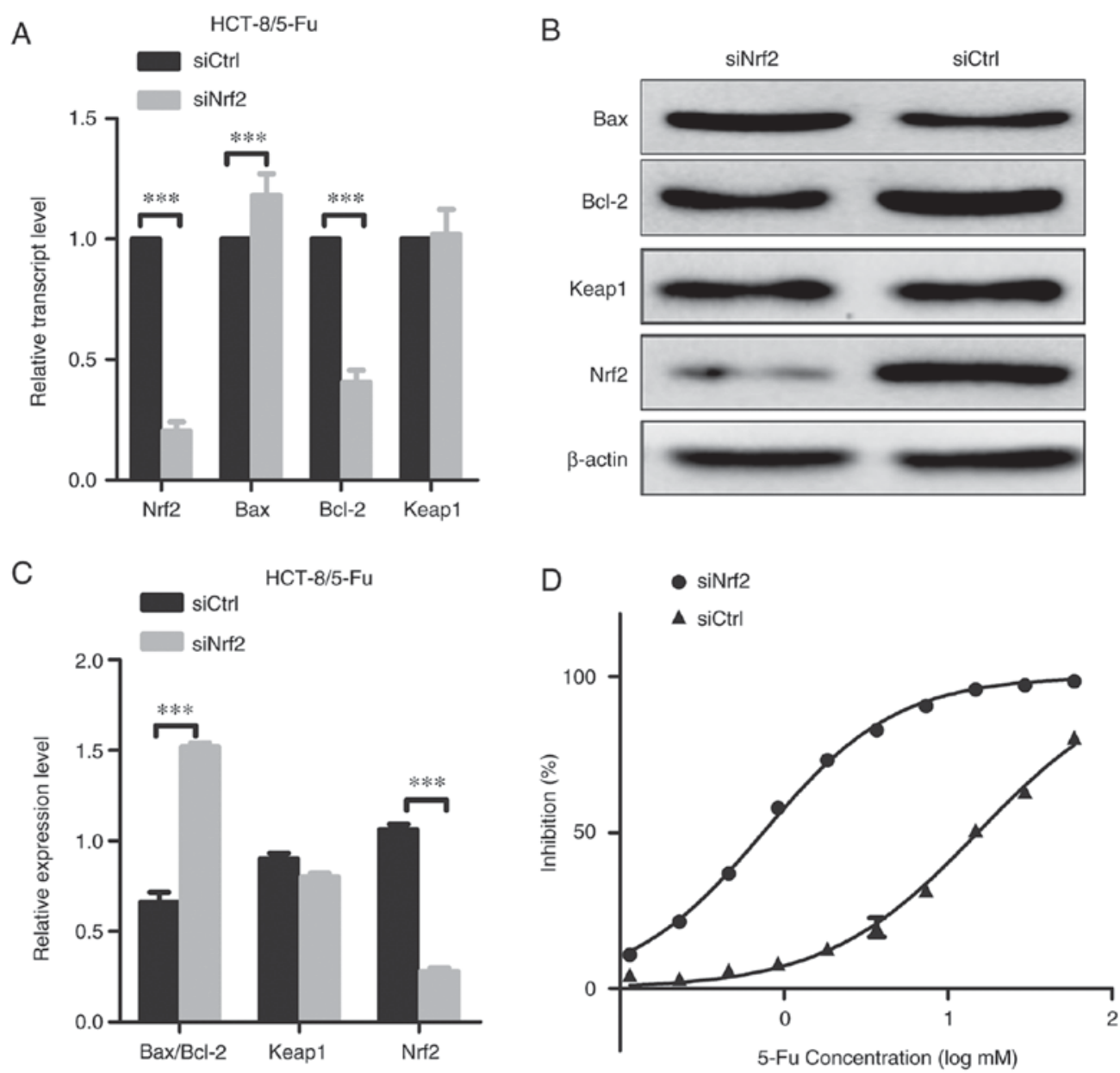

Figure 4. Effect of Nrf2 on the MDR reversal effect of curcumin in HCT-8/5-Fu cells. HCT-8/5-Fu cells were treated with siCtrl or siNrf2 for $48 \mathrm{~h}$. The effect on the mRNA and protein expression levels of Bax, Bcl-2, Keap1 and Nrf2 were evaluated by (A) reverse transcription-quantitative polymerase chain reaction analysis and (B) western blot analysis, respectively. (C) Relative protein expression levels were quantified using ImageJ and normalized to $\beta$-actin. Data are expressed as the mean \pm standard deviation of three independent experiments. ${ }^{* * *} \mathrm{P}<0.005$. (D) Cell viability was measured using a 3-(4,5-dimethyl-2-thiazol)-2,5-diphenyltetrazolium bromide assay. Nrf2, nuclear factor erythroid 2-related factor; MDR, multidrug resistance; si-, small interfering RNA; Ctrl, negative control; Bcl-2, B-cell lymphoma 2; Bax, Bcl-2-associated X protein; Keap1, Kelch like ECH associated protein 1.

results were obtained following western blot analysis of the protein levels (Fig. 3B and C). Furthermore, upregulation of the protein expression level of Nrf2 was observed in HCT-8/5-Fu cells following treatment with a gradient of curcumin concentrations $(1,10$ and $20 \mu \mathrm{M})$ compared with that in normal cells (Fig. 3E). A previous report demonstrated that Nrf2 was able to directly activate the transcription of the anti-apoptotic protein Bcl-2, whereas Keapl induced Bcl-2 ubiquitination via Cullin 3 (Cul3) and stabilized Bax $(21,22)$. The results of the present study indicated that curcumin significantly repressed the mRNA and protein expression levels of Nrf2 following combined treatment with 5-Fu in HCT-8/5-Fu cells, leading to reduced mRNA and protein expression levels of Bcl-2, which induced cell apoptosis further.

Nrf2 is a key factor in determining the MDR reversal effect of curcumin in HCT-8/5-Fu cells. To investigate whether Nrf2 has a functional role in determining the MDR reversal effect of curcumin in HCT-8/5-Fu cells, the present study first reduced the expression of Nrf2 at the mRNA and protein levels in the HCT-8/5-Fu cells using a siNrf2 (Fig. 4A and B), as in our previous report (23). The expression of $\mathrm{Bcl}-2$ was reduced at the mRNA and protein levels in cells treated with siNrf2 compared with cells transfected with siCtrl only, however, the expression of Keap1 was not altered and the expression level of Bax was increased (Fig. 4B and C). These results confirmed that Nrf2 directly promoted the expression of Bcl-2 at the mRNA and protein levels. Subsequently, the present study examined the cytotoxicity of $5-\mathrm{Fu}$ towards $\mathrm{HCT}-8 / 5-\mathrm{Fu}$ cells treated with siNrf2 or siRNA alone using MTT assays. The results revealed that cells treated with siNrf2 $\left(\mathrm{IC}_{50}: 0.7469 \pm 0.02321\right.$ $\mathrm{mM}$ ) were more sensitive to $5-\mathrm{Fu}$ than cells treated with siCtrl ( $\mathrm{IC}_{50}: 15.20 \pm 0.1362 \mathrm{mM}$; Fig. 4D). Taken together, these results indicated that the effect of siNrf2 combined with $5-\mathrm{Fu}$ treatment in HCT-8/5-Fu cells was similar to that observed in cells treated with curcumin combined with $5-\mathrm{Fu}$, leading to the reduced expression of Nrf2 at the mRNA and protein levels, which further suppressed the expression levels of $\mathrm{Bcl}-2$, thereby promoting apoptosis to reverse MDR.

\section{Discussion}

Chemotherapy with cytotoxic drugs is essential in cancer treatment and is often used following surgery as an adjuvant 
therapy in treating patients with advanced colon cancer (24). However, drug resistance develops in almost all patients with colon cancer, which leads to chemotherapy failure (3). Therefore, screening effective MDR reversal agents, and combining them with cytotoxic drugs has been a promising strategy to overcome MDR and thereby improve the therapeutic efficacies of MDR in cancer (25). Over several decades, compounds, including verapamil, cyclosporine, quinidine and tamoxifen, have exhibited MDR reversal activity in vitro. However, these compounds have limited clinical applications due to their high toxicity and low activity (25). A number of studies have reported that curcumin can reverse MDR by regulating the expression of genes involved in drug efflux transport and apoptosis in a variety of MDR cancer cells, and exhibit low toxicity and high activity (26-28). Therefore, a comprehensive understanding of the underlying mechanisms of curcumin is critical for the development and improvement of novel treatment strategies for patients with MDR.

A previous study suggested that curcumin may reverse the MDR of HCT-8/5-Fu cells by decreasing the expression levels of several genes involved in the drug efflux pump and in apoptosis, including p-glycoprotein, heat shock protein 27 , survivin, and Bcl-2 (29). However, the majority of the previous studies have focused on the regulatory effect of curcumin on the expression of Bcl-2 in human cancer cells, including colorectal cancer, hepatoma, renal carcinoma and glioblastoma cells (30-33). In the present study, the regulatory effect of curcumin in MDR cells was also investigated, and a significant increase in apoptosis was observed only in cells treated with curcumin and 5-Fu combined; treatment with curcumin or 5-Fu alone did not markedly induce apoptosis compared with that in the untreated control cells. To confirm these results, the expression of Bcl-2 and Bax at the mRNA and protein levels was determined; the expression ratio of $\mathrm{Bcl}-2 / \mathrm{Bax}$ was relatively unchanged in cells treated with curcumin or 5-Fu alone compared with that in untreated control cells. By contrast, this ratio was markedly reduced in cells treated with curcumin and 5-Fu in combination. Taken together, these results demonstrated that combined treatment with curcumin and $5-\mathrm{Fu}$ in HCT-8/5-Fu cells significantly decreased the expression ratio of $\mathrm{Bcl}-2 / \mathrm{Bax}$ compared with that in untreated control cells, which in turn increased the levels of apoptosis. However, treatment with curcumin alone in HCT-8/5-Fu cells did not induce apoptosis compared with that in untreated control cells as curcumin had no effect on the expression ratio of $\mathrm{Bcl}-2 / \mathrm{Bax}$.

A previous study described an important connection between the Keap1/Nrf2 signaling pathway and the Bcl-2 family. Keap1 directly binds to the $\mathrm{BH} 2$ domain of $\mathrm{Bcl}-2$ and induces its ubiquitination via $\mathrm{Cul} 3$ in a conserved lysine residue, which then triggers $\mathrm{Bcl}-2$ proteasomal degradation (21). In addition, Nrf2 is able to directly activate the transcription of the antiapoptotic proteins Bcl-2 and Bcl-extra large, resulting in increased cell survival and drug resistance $(19,22)$. Taken together, these results demonstrated that apoptosis is inhibited via the activation of Nrf2 and, in turn, high levels of functional Keap1 (and therefore inactive Nrf2) result in its induction by proapoptotic proteins. Therefore, the present study examined the expression levels of Nrf2 and other reference proteins between HCT- 8 and HCT-8/5-Fu cells. It was found that the expression of $\mathrm{Nrf} 2$ was upregulated in
HCT-8/5-Fu cells. Subsequently, the present study investigated the expression of Keap1 and Nrf2 at the mRNA and protein levels in HCT-8/5-Fu cells with or without treatment. The results revealed that the expression of $\mathrm{Nrf} 2$ was reduced in HCT-8/5-Fu cells treated with curcumin and 5-FU combined compared with that in the untreated cells, which was consistent with the results of the MTT assay. In addition, sensitivity to 5-Fu was increased in the HCT-8/5-Fu cells following knockdown of the expression of Nrf2. Therefore, Nrf2 may have a functional role in promoting the apoptosis of HCT-8/5-Fu cells via curcumin, which may be the underlying mechanism for the MDR reversal effect of curcumin in HCT-8/5-Fu cells. In addition, the increased sensitivity to $5-\mathrm{Fu}$ induced cytotoxicity in Nrf2-deficient HCT-8/5-Fu cells when compared with normal HCT-8/5-Fu cells, which further supported this conclusion.

In conclusion, the present study confirmed the reversal effect of curcumin on MDR in HCT-8/5-Fu cells and demonstrated for the first time, to the best of our knowledge, the favorable effect of $\mathrm{Nrf} 2$ in the MDR reversal effect of curcumin in colorectal cancer cells. Curcumin, at a non-cytotoxic dose, combined with $5-\mathrm{Fu}$ induced apoptosis by decreasing the expression of $\mathrm{Nrf2}$, which in turn suppressed the expression ratio of $\mathrm{Bcl}-2 / \mathrm{Bax}$, and consequently reversed the MDR of HCT-8/5-Fu cells. Therefore, the combination of curcumin with cytotoxic drugs may serve as a promising strategy for chemotherapy in patients with colorectal cancer.

\section{Acknowledgements}

Not applicable.

\section{Funding}

The present study was supported by the National Natural Science Foundation of Chin (grant nos. 81272485, 81641141 and 81503236), the Natural Science Foundation of Anhui Province (grant nos. 1808085QH262, 1608085QH212 and 1408085MH163), the University Natural Science Research Project of Anhui Province (grant no. KJ2018A0259), Provincial Foundation for Excellent Young Talents of Colleges and Universities of Anhui Province (grant nos. gxyqZD2016178 and gxyqZD2016179), Specialized Research Fund for the Doctoral Program of Wannan Medical College (grant no. rcqd201607), Special Foundation for Talents of Yijishan Hospital (grant nos. YR201407 and YR201501), Key Research Cultivation Foundation of Wannan Medical College (grant nos. WK2017Z03 and WK2015ZF01), Foundation for Wannan Medical College Academic and Technical Leaders and Foundation for the Back-Up of Wannan Medical College Academic and Technical Leaders.

\section{Availability of data and materials}

All data generated or analyzed during this study are included in this published article.

\section{Authors' contributions}

$\mathrm{CZ}$ performed the experiments, wrote the manuscript and analyzed the data. XPL designed the experiments and wrote 
the manuscript. LJH performed the MTT assays and wrote the manuscript. HZY performed the apoptosis and western blot assays. DFL performed the RNA interference assays. YBZ performed the reverse transcription-quantitative polymerase chain reaction assays. DDM and SPZ performed cell cultures. YYC and YWJ performed cell culture and drug treatment assays. JS performed quantification of protein expression levels from western blots using ImageJ software. All authors read and approved the final manuscript.

\section{Ethics approval and consent to participate}

Not applicable.

\section{Patient consent for publication}

Not applicable.

\section{Competing interests}

The authors declare that they have no competing interests.

\section{References}

1. Chen W, Zheng R, Baade PD, Zhang S, Zeng H, Bray F, Jemal A, Yu XQ and He J: Cancer statistics in China, 2015. CA Cancer J Clin 66: 115-132, 2016.

2. Cunningham D, Atkin W, Lenz HJ, Lynch HT, Minsky B, Nordlinger B and Starling N: Colorectal cancer. Lancet 375: 1030-1047, 2010

3. Dallas NA, Xia L, Fan F, Gray MJ, Gaur P, van Buren G II, Samuel S, Kim MP, Lim SJ and Ellis LM: Chemoresistant colorectal cancer cells, the cancer stem cell phenotype, and increased sensitivity to insulin-like growth factor-I receptor inhibition. Cancer Res 69: 1951-1957, 2009.

4. Kim JH, Chung JB, Park IS, Kim BS, Yoo NC, Choi JH, Roh JK, Kim HS, Kwon OH, Lee KS, et al: Combined use of tamoxifen, cyclosporin A, and verapamil for modulating multidrug resistance in human hepatocellular carcinoma cell lines. Yonse Med J 34: 35-44, 1993.

5. Thomas $\mathrm{H}$ and Coley HM: Overcoming multidrug resistance in cancer: An update on the clinical strategy of inhibiting p-glycoprotein. Cancer Contr 10: 159-165, 2003.

6. Ferry DR, Traunecker H and KerrDJ: Clinical trials of P-glycoprotein reversal in solid tumours. Eur J Cancer 32A: 1070-1081, 1996.

7. Lin SS, Lai KC, Hsu SC, Yang JS, Kuo CL, Lin JP, Ma YS, Wu CC and Chung JG: Curcumin inhibits the migration and invasion of human A549 lung cancer cells through the inhibition of matrix metalloproteinase-2 and -9 and Vascular Endothelial Growth Factor (VEGF). Cancer Lett 285: 127-133, 2009.

8. Limtrakul P: Curcumin as chemosensitizer. Adv Exp Med Biol 595: 269-300, 2007.

9. Sharma RA, Gescher AJ and Steward WP: Curcumin: The story so far. Eur J Cancer 41: 1955-1968, 2005.

10. Anand P, Thomas SG, Kunnumakkara AB, Sundaram C, Harikumar KB, Sung B, Tharakan ST, Misra K, Priyadarsini IK, Rajasekharan KN, et al: Biological activities of curcumin and its analogues (Congeners) made by man and Mother Nature. Biochem Pharmacol 76: 1590-1611, 2008.

11. Guzzarlamudi S, Singh PK, Pawar VK, Singh Y, Sharma K, Paliwal SK, Chourasia MK, Ramana MV and Chaurasia M: Synergistic Chemotherapeutic Activity of Curcumin Bearing Methoxypolyethylene Glycol-g-Linoleic Acid Based Micelles on Breast Cancer Cells. J Nanosci Nanotechnol 16: 4180-4190, 2016.

12. Colacino JA, McDermott SP, Sartor MA, Wicha MS and Rozek LS Transcriptomic profiling of curcumin-treated human breast stem cells identifies a role for stearoyl-coa desaturase in breast cancer prevention. Breast Cancer Res Treat 158: 29-41, 2016.

13. Muddineti OS, Kumari P, Ajjarapu S, Lakhani PM, Bahl R, Ghosh B and Biswas S: Xanthan gum stabilized PEGylated gold nanoparticles for improved delivery of curcumin in cancer. Nanotechnology 27: 325101, 2016.
14. Livak KJ and Schmittgen TD: Analysis of relative gene expression data using real-time quantitative PCR and the 2(-Delta Delta C(T)) method. Methods 25: 402-408, 2001.

15. Gottesman MM, Fojo T and Bates SE: Multidrug resistance in cancer: Role of ATP-dependent transporters. Nat Rev Cancer 2: 48-58, 2002.

16. Fernald $\mathrm{K}$ and Kurokawa M: Evading apoptosis in cancer. Trends Cell Biol 23: 620-633, 2013.

17. Chapman RS, Whetton AD, Chresta CM and Dive C: Characterization of drug resistance mediated via the suppression of apoptosis by Abelson protein tyrosine kinase. Mol Pharmacol 48: 334-343, 1995.

18. Chapman RS, Whetton AD and Dive C: The suppression of drug-induced apoptosis by activation of $\mathrm{v}-\mathrm{ABL}$ protein tyrosine kinase. Cancer Res 54: 5131-5137, 1994

19. Niture SK and Jaiswal AK: Nrf2 protein up-regulates antiapoptotic protein $\mathrm{Bcl}-2$ and prevents cellular apoptosis. J Biol Chem 287: 9873-9886, 2012.

20. Chen Q, Li W, Wan Y, Xia X, Wu Q, Chen Y, Lai Z, Yu C and Li W: Amplified in breast cancer 1 enhances human cholangiocarcinoma growth and chemoresistance by simultaneous activation of Akt and Nrf2 pathways. Hepatology 55: 1820-1829, 2012.

21. Yin XM, Oltvai $\mathrm{ZN}$ and Korsmeyer SJ: $\mathrm{BH} 1$ and $\mathrm{BH} 2$ domains of $\mathrm{Bcl}-2$ are required for inhibition of apoptosis and heterodimerization with Bax. Nature 369: 321-323, 1994.

22. Niture SK and Jaiswal AK: Nrf2-induced antiapoptotic Bcl-xL protein enhances cell survival and drug resistance. Free Radic Biol Med 57: 119-131, 2013

23. Zhang C, Wang HJ, Bao QC, Wang L, Guo TK, Chen WL, $\mathrm{Xu}$ LL, Zhou HS, Bian JL, Yang YR, et al: NRF2 promotes breast cancer cell proliferation and metastasis by increasing RhoA/ROCK pathway signal transduction. Oncotarget 7 : 73593-73606, 2016.

24. Stein A, Atanackovic D and Bokemeyer C: Current standards and new trends in the primary treatment of colorectal cancer. Eur J Cancer 47 (Suppl 3): S312-S314, 2011.

25. Robert J and Jarry C: Multidrug resistance reversal agents. J Med Chem 46: 4805-4817, 2003.

26. Wang J, Wang F, Li F, Zhang W, Shen Y, Zhou D and Guo S: A multifunctional poly(curcumin) nanomedicine for dual-modal targeted delivery, intracellular responsive release, dual-drug treatment and imaging of multidrug resistant cancer cells. J Mater Chem B Mater Biol Med 4: 2954-2962, 2016.

27. Baldwin PR, Reeves AZ, Powell KR, Napier RJ, Swimm AI, Sun A, Giesler K, Bommarius B, Shinnick TM, Snyder JP, et al: Monocarbonyl analogs of curcumin inhibit growth of antibiotic sensitive and resistant strains of Mycobacterium tuberculosis. Eur J Med Chem 92: 693-699, 2015.

28. Negi N, Prakash P, Gupta ML and Mohapatra TM: Possible Role of Curcumin as an Efflux Pump Inhibitor in Multi Drug Resistant Clinical Isolates of Pseudomonas aeruginosa. J Clin Diagn Res 8: DC04-DC07, 2014.

29. Fan YX, Abulimiti P, Zhang HL, Zhou YK and Zhu L: Mechanism of reversal of multidrug resistance by curcumin in human colorectal cancer cell line HCT-8/5-FU. Genet Mol Res 16: 16, 2017.

30. Seo BR, Min KJ, Cho IJ, Kim SC and Kwon TK: Curcumin significantly enhances dual PI3K/Akt and mTOR inhibitor NVP-BEZ235-induced apoptosis in human renal carcinoma Caki cells through down-regulation of p53-dependent Bcl-2 expression and inhibition of Mcl-1 protein stability. PLoS One 9: e95588, 2014.

31. Sun Y, Zhang J, Zhou J, Huang Z, Hu H, Qiao M, Zhao X and Chen D: Synergistic effect of cucurbitacin B in combination with curcumin via enhancing apoptosis induction and reversing multidrug resistance in human hepatoma cells. Eur J Pharmacol 768: 28-40, 2015.

32. Moghadamtousi SZ, Kadir HA, Hassandarvish P, Tajik H, Abubakar S and Zandi K: A review on antibacterial, antiviral, and antifungal activity of curcumin. Biomed Res Int 2014: $186864,2014$.

33. Patel BB and Majumdar AP: Synergistic role of curcumin with current therapeutics in colorectal cancer: Minireview. Nutr Cancer 61: 842-846, 2009.

This work is licensed under a Creative Commons Attribution-NonCommercial-NoDerivatives 4.0 International (CC BY-NC-ND 4.0) License. 